\title{
Seuils de stabilité pour un écoulement à surface libre engendré dans une cavité cylindrique tournante à petit rapport de forme
}

\author{
Lyes Kahouadji $^{1,2, a}$, Laurent Martin Witkowski ${ }^{1,2}$ et Patrick Le Quéré ${ }^{1}$ \\ 1 CNRS, UPR 3251, LIMSI, 91403 Orsay, France \\ 2 UPMC, 4 place Jussieu, 75005 Paris, France
}

Reçu le 25 janvier 2010, accepté le 28 septembre 2010

\begin{abstract}
Résumé - Par une étude de stabilité linéaire nous nous proposons d'analyser un écoulement dans une cavité cylindrique remplie de fluide, dont le fond tourne. Le rayon de la cavité est grand devant la hauteur de fluide. Une étude expérimentale récente [S. Poncet, M.P. Chauve, J. Flow Vis. Image Process 14 (2007) 85-105] a mis en évidence une instabilité aux motifs particulièrement intéressants. Dans ce travail, nous considérons une modélisation de la surface libre plane (conditions de symétrie) afin de comparer les résultats expérimentaux et notre étude de stabilité linéaire. Nous discuterons de la pertinence de cette modélisation, c'est-à-dire de la nécessité de prendre en compte la déformation de la surface.
\end{abstract}

Mots clés : Écoulement tournant / stabilité linéaire

\begin{abstract}
Stability threshold for the free surface flow driven by a rotating disk in a small aspect ratio cylindrical cavity. We perform a linear stability analysis of the flow inside a cylindrical tank in which the bottom rotates. The aspect ratio is such that the radius of the disk is larger than the fluid height. In a recent experiment [S. Poncet, M.P. Chauve, J. Flow Vis. Image Process 14 (2007) 85-105] interesting instability patterns have been found. As the fluid rotates, the free surface is deformed, which increases the computational difficulty. As a first approach, we consider a non deformable free surface (symmetry condition). We discuss the validity of this hypothesis by comparing our results with the experiments. Finally, we discuss the necessity to take into acount the free surface deformation.
\end{abstract}

Key words: Rotating flow / linear stability

\section{Introduction}

Les écoulements en cavité cylindrique, plus précisément les écoulements inter-disques, ont été grandement étudiés dans la littérature en tant que configuration modèle. Ils reproduisent de nombreuses situations d'intérêt géophysique ou industriel. Les écoulements en turbomachines ou le refroidissement des disques durs d'ordinateur peuvent en particulier se modéliser par différents types d'écoulements inter-disques.

Plusieurs études ont été réalisées dans le cadre d'un fluide confiné entre deux plaques avec différentes configurations (rotor-stator, contra-rotation, ECDP ${ }^{1}$, etc.). Dans ces écoulements, des motifs polygonaux intrigants ont été expérimentalement observés et retrouvés numériquement par de nombreux auteurs [1,2]. Dans le cas des écoulements de type Contra-Rotation,

\footnotetext{
a Auteur pour correspondance : lyes@limsi.fr

1 Enclosed corotating disk pair.
}

Martin Witkowski et al. [3] ont trouvé, par une étude numérique et par un développement asymptotique, que les instabilités axi-symétriques sont d'origine centrifuge. Des travaux comme ceux de Nore et al. [4] ont montré numériquement et expérimentalement, dans la même configuration et pour différents rapports de forme, qu'il y a une compétition entre les instabilités du mode axisymétrique et des instabilités de type 3D. Des motifs polygonaux ont été observés pour différents paramètres (géométrie des cavités, vitesses angulaires des disques). Ces motifs sont formés d'un nombre fini $m$ de vortex. Niino et Misawa [5] ont mis en évidence que $m$ décroît quand le nombre de Reynolds augmente. Chomaz et al. [1] ont montré expérimentalement et numériquement que $m$ dépend non seulement du nombre de Reynolds mais aussi du rapport de forme. Le mécanisme de cette instabilité est attribué à un cisaillement de type Kelvin Helmholtz. Notre étude est purement numérique et s'appuie sur des travaux expérimentaux de Poncet et Chauve [6] (voir le 


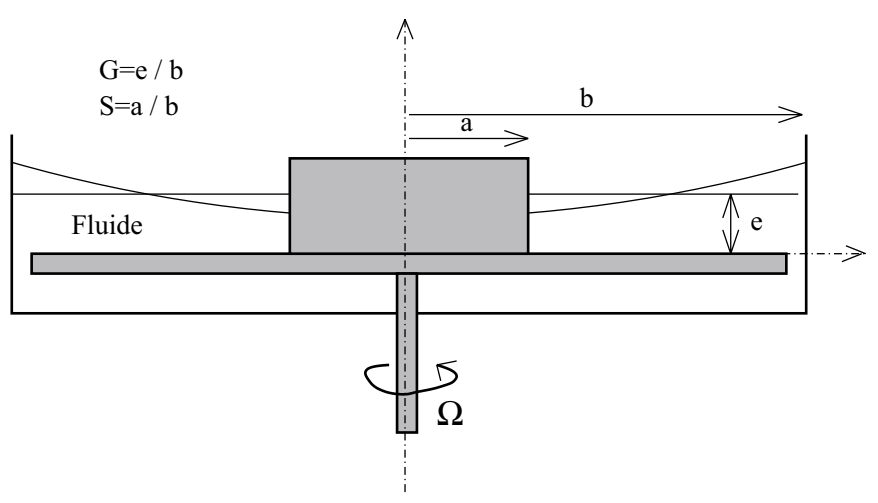

(a)

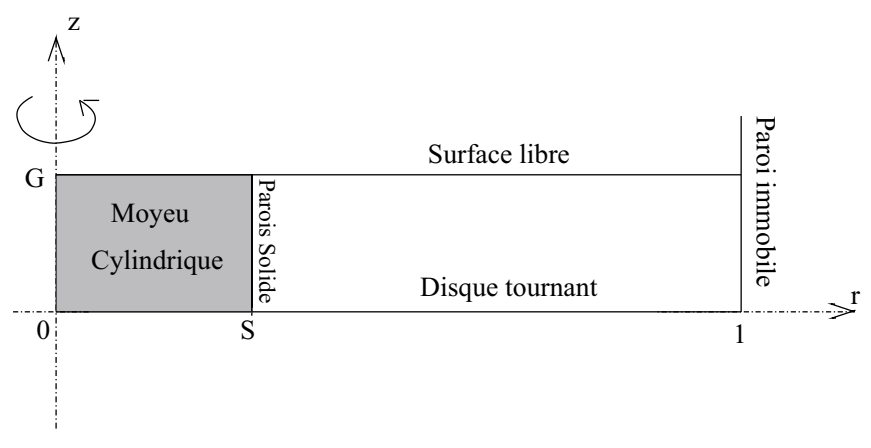

(b)

Fig. 1. (a) Schéma du dispositif expérimental [6]; (b) modèle utilisé.

dispositif dans Fig. 1a). Nous discuterons de la pertinence d'une modélisation où la surface libre reste plane. La première partie traite la formulation du problème en posant toutes les équations et les conditions aux limites de l'écoulement de base et de perturbation. Nous discuterons ensuite des résultats numériques tout en les comparant avec les expériences de Poncet et Chauve [6]. À la fin, nous conclurons par la critique de nos hypothèses et des perspectives à envisager.

\section{Modélisation}

Le dispositif expérimental étudié dans [6] est schématisé en figure 1. Un disque de rayon extérieur $b$ auquel est éventuellement fixé un moyeu de rayon $a$ tourne à la vitesse angulaire $\Omega$ dans une cavité fixe de rayon intérieur $b$. Dans cette cuve ainsi constituée est mis un liquide supposé incompressible, de masse volumique $\rho$ et de viscosité cinématique $\nu$. La hauteur du liquide au-dessus du disque tournant est $e$. On note $G=e / b$ le rapport de forme vertical de l'espace occupé par le liquide et $S=a / b$ le rapport des rayons. Si la vitesse angulaire $\Omega$ reste faible, on peut négliger la variation de la hauteur de liquide et on considérera en première approximation que l'écoulement se produit dans le domaine représenté sur la figure $1 \mathrm{~b}$.

Les équations du mouvement sont mises sous forme adimensionnelle en utilisant $b$ comme longueur de référence et $1 / \Omega$ comme temps de référence. La vitesse de référence est alors $\Omega b$ et on prend $\rho(\Omega b)^{2}$ comme pression de référence.

\section{1 Écoulement de base}

L'écoulement de base, avant la première bifurcation, est stationnaire et axisymétrique. L'axisymétrie permet d'introduire deux fonctions scalaires, la vorticité $\omega$ et la fonction de courant $\psi$ pour le champ de vitesse méridien. Les composantes $\left(U_{r}, U_{\theta}, U_{z}\right)$ du champ de vitesse s'ex- priment à l'aide des relations :

$U_{r}=\frac{1}{r} \frac{\partial \psi}{\partial z}, U_{z}=-\frac{1}{r} \frac{\partial \psi}{\partial r}, \omega=\frac{\partial U_{r}}{\partial z}-\frac{\partial U_{z}}{\partial r}$ et $\Gamma=r \cdot U_{\theta}$

où $\Gamma$ est le moment angulaire.

Le choix de cette formulation permet d'éliminer la pression dans les équations de Navier-Stokes et d'économiser une équation à résoudre. Le système d'équations gouvernant l'écoulement de base se réduit à :

$$
\frac{\partial^{2} \psi}{\partial r^{2}}-\frac{1}{r} \frac{\partial \psi}{\partial r}+\frac{\partial^{2} \psi}{\partial z^{2}}=r \omega
$$

$$
\begin{aligned}
\frac{\partial}{\partial r}\left(U_{r} \omega\right)+\frac{\partial}{\partial z}\left(U_{z} \omega\right)-\frac{\partial}{\partial z}\left(\frac{\Gamma^{2}}{r^{3}}\right)= & \frac{1}{R e}\left(\frac{\partial^{2} \omega}{\partial r^{2}}+\frac{1}{r} \frac{\partial \omega}{\partial r}\right. \\
& \left.-\frac{\omega}{r^{2}}+\frac{\partial^{2} \omega}{\partial z^{2}}\right)
\end{aligned}
$$

$\frac{\partial}{\partial r}\left(U_{r} \Gamma\right)+\frac{U_{r} \Gamma}{r}+\frac{\partial}{\partial z}\left(U_{z} \Gamma\right)=\frac{1}{R e}\left(\frac{\partial^{2} \Gamma}{\partial r^{2}}-\frac{1}{r} \frac{\partial \Gamma}{\partial r}+\frac{\partial^{2} \Gamma}{\partial z^{2}}\right)$

où $R e=\Omega b^{2} / \nu$ est le nombre de Reynolds défini par la rotation du disque. Les conditions aux limites ${ }^{2}$ sont :

Condition ouest : si $r=S=0, \psi=\omega=\Gamma=0$, sinon

$$
\text { pour } r=S \neq 0, \psi=0, \omega=\frac{1}{r} \frac{\partial^{2} \psi}{\partial r^{2}} \text { et } \Gamma=S^{2}
$$

Condition est (la paroi fixe) $: r=1: \psi=0$,

$$
\omega=\frac{1}{r} \frac{\partial^{2} \psi}{\partial r^{2}} \text { et } \Gamma=0
$$

Condition nord (la surface libre) $: z=G$ :

$$
\psi=\omega=\frac{\partial \Gamma}{\partial z}=0
$$

${ }^{2}$ Il est noté que le cas $S=0$ n'a pas les mêmes conditions que le cas $S \neq 0$. 


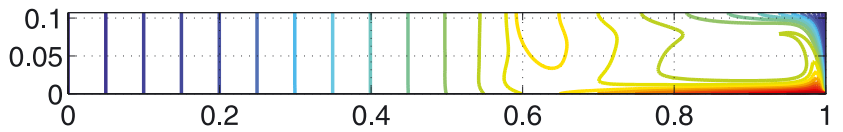

$\left(\mathrm{a}_{1}\right)$

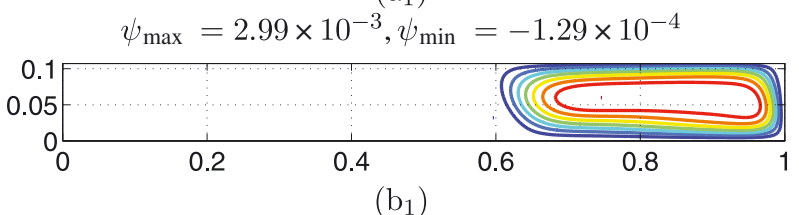

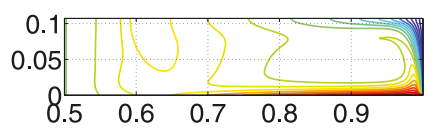

$\left(\mathrm{a}_{2}\right)$

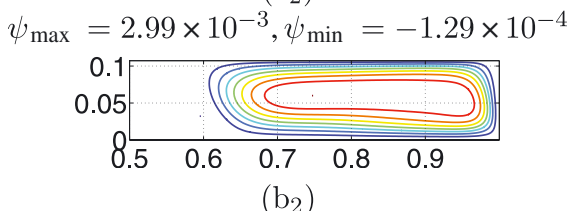

Fig. 2. Pour $G=0,107$ et $R e=10017\left(\mathrm{a}_{1}, \mathrm{a}_{2}\right)$ : champ de vitesse $U_{\theta}$, $\left(\mathrm{b}_{1}\right.$ et $\left.\mathrm{b}_{2}\right)$ : iso-contour de $\psi$. ( $\grave{\mathrm{A}}$ gauche, cavité correspondant à $S=0$, à droite $S=0,5)$.

Condition sud (le disque tournant) : $z=0: \psi=0$

$$
\omega=\frac{1}{r} \frac{\partial^{2} \psi}{\partial z^{2}} \text { et } \Gamma=r^{2}
$$

\subsection{Perturbation}

Le champ de vitesse est décomposé de la manière suivante :

$$
\begin{aligned}
\mathbf{V}(r, \theta, z, t)=\mathbf{U}(r, z)+\epsilon \operatorname{Real}\left[\left(v_{r}(r, z),\right.\right. \\
\\
\left.\left.\quad i v_{\theta}(r, z), v_{z}(r, z)\right) \exp (\lambda t+i m \theta)\right]
\end{aligned}
$$

où $\epsilon$ est un petit paramètre, $m$ est le nombre d'onde azimutal et $\lambda=\lambda_{r}+i \lambda_{i}$ définit le taux de croissance $\lambda_{r}$ et la fréquence $\lambda_{i}$. Les variables $v_{r}, v_{\theta}, v_{z}$ sont complexes. Une formulation similaire est utilisée pour la perturbation de pression $p$. En utilisant la décomposition (9), les équations de Navier-Stokes à l'ordre $\epsilon$ sont identiques aux équations exprimées dans Kahouadji et al. $[7]^{3}$. Les conditions aux limites s'écrivent :

Pour $r=1$ ou $z=0: v_{r}=v_{\theta}=v_{z}=0$

(condition d'adhérence à la paroi)

Pour $z=G: v_{z}=\frac{\partial v_{\theta}}{\partial z}=\frac{\partial v_{r}}{\partial z}=0$

(condition de symétrie à la surface libre)

Pour $r=S=0: \frac{\partial v_{\theta}}{\partial r}=\frac{\partial v_{r}}{\partial r}=v_{z}=0$, pour $m=1$,

$$
v_{r}=v_{\theta}=v_{z}=0 \text { pour } m>1
$$

et $v_{r}=v_{\theta}=\frac{\partial v_{z}}{\partial r}=0$ pour $m=0$ (condition sur l'axe)

pour $r=S \neq 0: v_{r}=v_{\theta}=v_{z}=0$

(condition d'adhérence au moyeu)

\footnotetext{
${ }^{3}$ La décomposition est similaire à celle utilisée dans cet article, les équations de Navier-Stokes demeurent inchangées sans prendre en compte l'équation de la thermique (8 à 11).
}

\subsection{Discrétisation et résolution numérique}

La discrétisation numérique est effectuée sur un maillage de $N R \times N Z$ points uniformément répartis dans chaque direction $r$ et $z$. Nous avons utilisé un schéma aux différences finies d'ordre 2 pour discrétiser les équations de l'écoulement de base et de perturbation.

La recherche des seuils critiques $R e_{\mathrm{c}}$ en fonction du rapport de forme $G$ se fait à l'aide des étapes suivantes :

- Pour Re et $G$ fixés, l'écoulement de base est résolu par une méthode de Newton (le système linéaire est résolu par LAPACK ${ }^{4}$ pour chaque itération de Newton).

- Les valeurs propres $\lambda$ sont trouvées à l'aide de la librairie ARPACK ${ }^{5}$.

- Avec une sécante sur $\lambda_{r}, R e_{\mathrm{c}}$ est trouvé quand $\lambda_{r} \approx 0$.

\section{Résultats}

\subsection{Structure de l'écoulement de base}

Pour différentes valeurs de $G \ll 1$ et pour $R e \sim R e_{\mathrm{c}}$, l'allure de l'écoulement de base est semblable à celle représentée sur la figure 2 qui montre respectivement le champ de vitesse azimutale $U_{\theta}\left(2 \mathrm{a}_{1}\right)$ et la fonction de courant $\psi\left(2 b_{1}\right)$. Ces figures montrent que dans une région proche de l'axe, le fluide est en rotation solide. Cela se remarque sur la figure $2 \mathrm{a}_{1}$, où les iso-contours de $U_{\theta}$ sont des droites verticales régulièrement espacées dans la direction radiale, sur la figure $2 \mathrm{~b}_{1}$, où la fonction de courant $\psi$ est très faible, signifiant l'absence de recirculation. Cette région s'étend radialement quand $G$ diminue. La rotation solide a été observée expérimentalement par Poncet et Chauve [6] et numériquement par Herrero et al. [8] et Randriamampianina et al. [9].

Ceci explique que la présence d'un moyeu n'affecte pas l'écoulement de base tant que $S \leqslant 0,5$. La structuration de l'écoulement obtenu pour $S=0,5$ est représentée sur les figures $2 \mathrm{a}_{2}, \mathrm{~b}_{2}$. On constate un excellent accord qualitatif, et des comparaisons quantitatives montrent que les valeurs maximales de $\omega$ et $\psi$ ne diffèrent que de $0,002 \%$. De plus, comme cela est discuté dans la section suivante, la présence du moyeu ne modifie pas les instabilités dans la zone cisaillée tant que $S \leq 0,5$.

\footnotetext{
4 Linear algebra package.

5 Arnoldi package.
} 


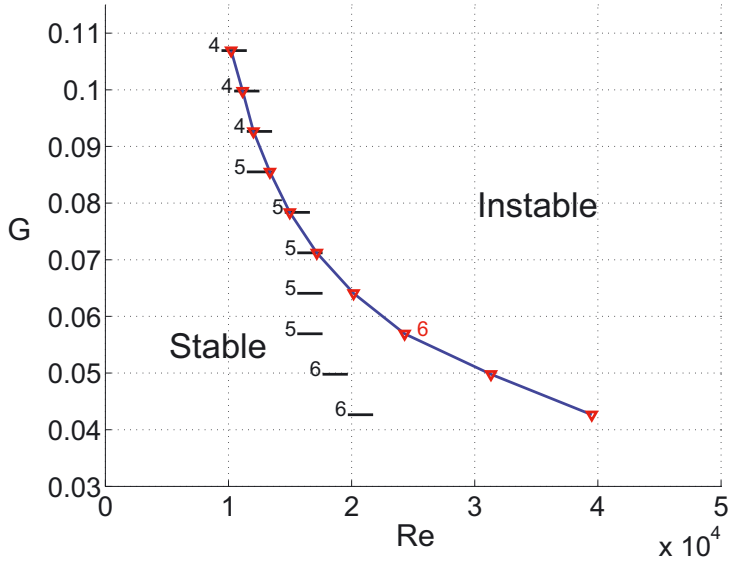

Fig. 3. Courbe de stabilité : les symboles «— désignent les valeurs expérimentales de Poncet et Chauve; la courbe continue marquée par $« ₫$ représente les résultats numériques (pour $S=0$ ); les modes azimutaux $m$ sont indiqués pour les valeurs expérimentales (et pour les valeurs numériques en cas de divergence).

\subsection{Analyse de stabilité linéaire}

La figure 3 représente l'évolution de $R e_{\mathrm{c}}$ en fonction de $G$. Les valeurs correspondantes sont reportées dans le tableau 1. Nous remarquons que $R e_{\mathrm{c}}$ croît lorsque $G$ décroît. Les modes azimutaux $m$ obtenus numériquement coïncident avec les expériences sur toute la plage des $G$ étudiés sauf pour $G \approx 0,057$. Le mode le plus instable est alors $m=6$. Cependant, en effectuant le calcul avec $m=5, R e_{\mathrm{c}}$ est égal à 24114 , les valeurs critiques de ces deux modes sont donc très proches. Les erreurs par rapport aux expériences sont comprises entre $2,3 \%$ et $6,8 \%$ quand $G \geqslant 0,071$. En dessous de cette valeur, les erreurs sont supérieures à $20 \%$ et dépassent même $90 \%$ lorsque $G=0,043$.

La figure 4 montre que l'étude de stabilité linéaire semble capter le lieu des instabilités. Le fort gradient qu'on observe se situe, pour toutes les valeurs de $G$, dans une région proche de la frontière entre la recirculation méridienne et la rotation solide, ce qui indique que l'instabilité semble due au cisaillement azimutal entre le fluide.

Les calculs ont également été effectués en présence d'un moyeu $(S=0,5)$. Le pas d'espace est le même que celui utilisé dans tout le domaine ce qui est obtenu en divisant par deux le nombre de points dans la direction radiale. Les valeurs de $R e_{\mathrm{c}}$ pour les modes les plus instables sont proches de celles trouvées dans tout le domaine à $1.7 \%$ près. Comme dans le cas sans moyeu, pour $G \sim 0,057$, le mode azimutal diffère de celui trouvé expérimentalement. Il faut cependant noter que $R e_{\mathrm{c}}=24240$ pour $m=5$. Cela montre encore une fois que la transition entre un mode 5 et un mode 6 a probablement lieu pour un rapport de forme très proche de 0,057. En revanche, lorsque le moyeu est plus grand que la zone de rotation solide, les instabilités semblent d'une autre nature. Ceci est observé expérimentalement, comme indiqué par Poncet et Chauve ([6], Fig. 16). En fixant
$G=0,0429$ et $R e=36945$ avec trois valeurs du rapport des rayons $S=0,286,0,536$ et $S=0,75$, ils ont montré que pour $S=0,75$, les structures des instabilités sont profondément modifiées. Bien que la valeur de $R e_{\mathrm{c}}$ soit très différente, nous avons pu reproduire les structures de l'instabilité (obtenues à $S=0,286$ et $S=0,536$ ) en fixant $S=0,5$. En revanche, à $S=0,75$, nous n'avons pas reproduit les structures observées expérimentalement.

\section{Discussion}

Les écarts importants entre les seuils trouvés expérimentalement et nos valeurs numériques nous amènent à penser que certaines hypothèses de notre modèle sont trop fortes. Le nombre de Froude $\mathrm{Fr}=$ $\Omega^{2} b / g$ ( $g$ est la gravité) mesure le rapport entre les forces d'inertie et la force de gravité. On constate qu'au seuil le nombre de Froude augmente quand le rapport de forme $G$ diminue. Cela implique une plus grande déformation relative de la surface libre à petit $G$. En connaissant le champ de vitesse et de pression, il est possible de déterminer, une approximation de la hauteur du fluide $h(r)$. En écrivant au niveau de la surface libre $(z=G)$, que la composante normale du tenseur des contraintes $\underline{\underline{\sigma}}$ devrait être nulle :

$$
\mathbf{n} \cdot \underline{\underline{\sigma}} \cdot \mathbf{n}=0
$$

où $\mathbf{n}$ représente la normale à la surface (dans notre cas, le vecteur $e_{z}$ ), on obtient une estimation de $h(r)$ par :

$$
\frac{h}{F r}=p-\frac{2}{R e} \frac{\partial U_{z}}{\partial z}
$$

où $p$ est la pression, relation déjà présentée dans [10] (Eq. (17)). Nos calculs de hauteur de surface libre pour $G=1$ et $R e=900$, qui ne sont pas présentés ici, sont en très bon accord avec ceux correspondant à $\mathrm{D}=1$ ([10], Fig. 14).

Pour deux rapports de forme $G=0,1$ et $G=0,05$ et les valeurs de Re et $F r$ prises au seuil de l'apparition de l'instabilité dans l'expérience, nous avons représenté sur la figure 5 la hauteur de la surface libre calculée à l'aide de l'expression (15). Sur la même figure, la parabole

$$
h_{\text {para }}(r)=G+\frac{F r}{2}\left(r^{2}-\frac{1}{2}\right)
$$

correspondant à une rotation solide est aussi tracée (en trait fin). Il y a un très bon accord sur la courbure $h^{\prime \prime}(r)$ qui est donnée par $F r$ à $0,1 \%$ près dans la zone $r<r_{\text {para }}$, avec $r_{\text {para }} \sim 0,51$ et 0,7 pour $G=0,1$ et $G=0,05$ respectivement. Pour $r>r_{\text {para }}$, la présence de la paroi fixe entraîne une décroissance de $U_{\theta}^{2} / r$ et en conséquence de $\partial p / \partial r$. La pression, terme dominant dans le membre de droite de la relation (15), croît ainsi moins vite qu'une parabole. Il en résulte un déficit de fluide en périphérie par rapport à une rotation solide, qui explique, par conservation de la masse, le décalage entre les courbes $h$ et $h_{\text {para }}$ dans la région proche de l'axe. Le deuxième enseignement de la figure 5 porte sur l'amplitude de la déformation. 
Tableau 1. Valeurs numériques $R e_{\mathrm{c}}$ avec et sans moyeu, et expérimentales $R e_{\mathrm{c}}(\exp )$ pour les modes les plus instables en fonction de $G$ (hauteur du fluide/rayon du disque en $\mathrm{cm}$ ). La dernière colonne représente les écarts par rapport aux résultats expérimentaux. Les valeurs de $R e_{\mathrm{c}}(\exp )$ nous ont été communiquées par S. Poncet.

\begin{tabular}{ccccccc}
\hline $\mathrm{NR} \times \mathrm{NZ}(S=0)$ & $G=e / b$ & $m$ & $\operatorname{Re}_{\mathrm{c}}(S=0)$ & $\operatorname{Re}_{\mathrm{c}}(S=0,5)$ & $\operatorname{Re}_{\mathrm{c}}(\exp )(S=0)$ & écart en $\%$ \\
\hline $1214 \times 51$ & $0,6 / 14 \sim 0,043$ & 6 & - & 39081 & 20525 & 90,5 \\
$1020 \times 51$ & $0,7 / 14 \sim 0,05$ & 6 & - & 30035 & 18473 & 68,4 \\
$894 \times 51$ & $0,8 / 14 \sim 0,057$ & $6-6-5$ & 24112 & 24128 & 16420 & 46,8 \\
$796 \times 51$ & $0,9 / 14 \sim 0,064$ & 5 & 19966 & 20050 & 16420 & 21,5 \\
$718 \times 51$ & $1,0 / 14 \sim 0,071$ & 5 & 16976 & 17042 & 16420 & 3,3 \\
$653 \times 51$ & $1,1 / 14 \sim 0,079$ & 5 & 14778 & 14825 & 15394 & 4,0 \\
$600 \times 51$ & $1,2 / 14 \sim 0,086$ & 5 & 13163 & 13172 & 12315 & 6,8 \\
$554 \times 51$ & $1,3 / 14 \sim 0,093$ & 4 & 11831 & 12040 & 12315 & 3,9 \\
$501 \times 51$ & $1,4 / 14 \sim 0,1$ & 4 & 10957 & 10961 & 11289 & 2,9 \\
$476 \times 51$ & $1,5 / 14 \sim 0,107$ & 4 & 10017 & 10169 & 10263 & 2,3 \\
\hline
\end{tabular}

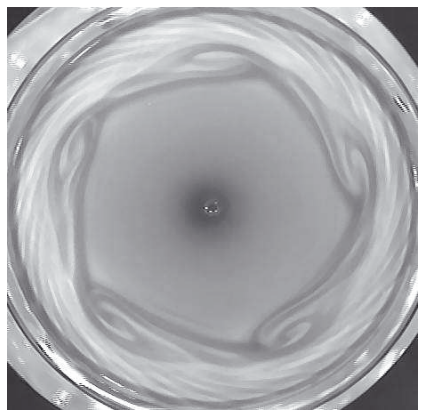

(a)

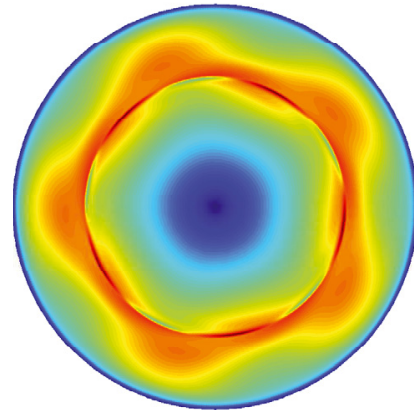

(b)
Fig. 4. $R e=24630, G=0,0714$ et $m=5$, (a) Visualisation de Poncet et Chauve, (b) champ de vitesse azimutale couplé avec les perturbations normalisées à la surface libre $\left(U_{\theta}+\epsilon \operatorname{Real}\left[i v_{\theta} / \max \left(\left\|v_{\theta}\right\|\right) \exp (i 5 \theta)\right]\right.$, avec $\epsilon$ arbitrairement choisi à 0,15$)$. La visualisation est obtenue pour $R e>R e_{\mathrm{c}}$ mais elle diffère peu de ce qui est observé au seuil (communication privée de Poncet).

Elle passe d'un peu moins de $2 \%$ pour $G=0,1$ à un peu plus de $10 \%$ pour $G=0,05$. L'amplitude relative de la déformation décroît donc avec $G$ et est proportionnelle à $\operatorname{Fr} /(2 G)$ (Éq. (16)).

\section{Conclusion}

Ce travail a été consacré à l'étude numérique d'un écoulement stationnaire et axisymétrique, engendré par un fond tournant d'une cavité cylindrique sous l'hypothèse d'une surface libre supposée plane. Il a été montré que pour des petits rapports de forme, la région proche de l'axe est en rotation solide et tourne exactement à la vitesse angulaire du fond. L'étude de stabilité linéaire de cet écoulement de base, dans la même hypothèse, a permis de retrouver qualitativement les valeurs des modes azimutaux et le lieu des instabilités observées expérimentalement. Cependant, si les valeurs de $R e_{\mathrm{c}}$ sont en bon accord avec les expériences pour des cuves suffisamment épaisses $(0,071 \leqslant G \leqslant 0,107)$, les écarts augmentent pour $G \leq 0,064$ et sont de l'ordre de $20 \%$ à $90 \%$. Dans le but de quantifier les effets de la surface

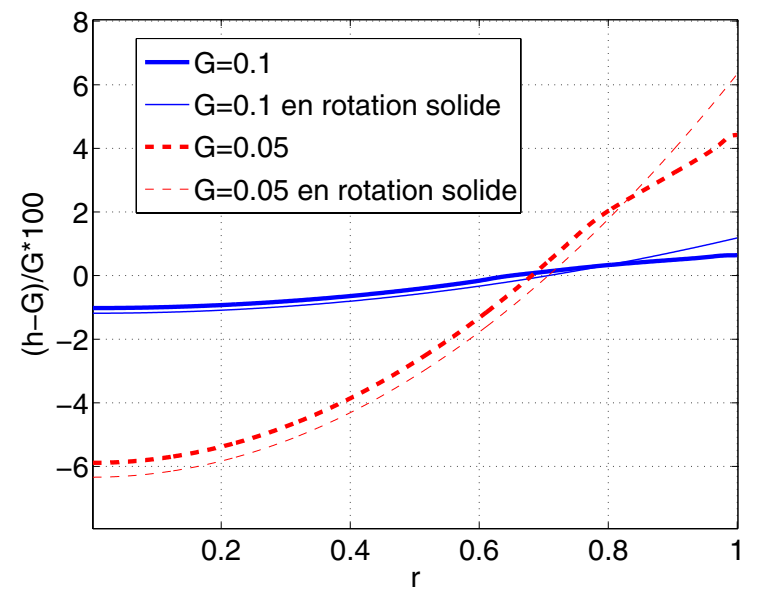

Fig. 5. Variation de la surface libre pour $(G, R e, F r)=$ $\left(0,1,11289,4,73 \times 10^{-3}\right)$ et $\left(0,05,18473,1,3 \times 10^{-2}\right)$.

libre, l'allure de cette dernière en fonction du rayon a été calculée en première approximation, montrant que la variation relative de la hauteur de fluide augmente quand le rapport de forme diminue. Cette constatation est une piste pour remettre en cause l'hypothèse de surface libre plane et ouvre la voie à de nouvelles investigations. En effet, il reste à expliquer comment une variation d'amplitude modérée, de l'ordre de $10 \%$, peut entraîner un écart sur le Reynolds critique de plus de $90 \%$. Nous nous proposons de vérifier si la remise en cause de l'hypothèse de surface libre est de nature à expliquer cette sensibilité sur la valeur du Reynolds critique.

Remerciements. Nous avons eu des discussions fructueuses avec O. Daube et S. Poncet. Nous les remercions chaleureusement.

\section{Références}

[1] J.M. Chomaz, M. Rabaud, C. Basdevant, Y. Couder, Experimental and numerical investigation of a forced circular shear layer, J. Fluid Mech. 187 (1988) 115-140 
[2] F. Moisy, O. Doaré, T. Pasutto, O. Daube, M. Rabaud, Experimental and numerical study of the shear layer instability between two counter-rotating disks, J. Fluid Mech. 507 (2004) 175-202

[3] L. Martin Witkowski, I. Delbende, P. Le Quéré, J.S. Walker, Axisymmetric stability of the flow between two exactly counter-rotating disks with large aspect ratio, J. Fluid Mech. 546 (2006) 193-202

[4] C. Nore, L. Martin Witkowski, E. Foucault, J. Pécheux, O. Daube, P. Le Quéré, Competition between axisymmetric and three-dimensional patterns between exactly counter-rotating disks, Phys. Fluids 18 (2006) 054102

[5] H. Niino, N. Misawa, An experimental and theoretical study of barotropic instability, J. Atmos. Sci. 41 (1984) 1992-2011
[6] S. Poncet, M.P. Chauve, Shear-layer instability in rotating system, J. Flow Vis. Image Process. 14 (2007) 85-105

[7] L. Kahouadji, L. Martin Witkowski, J.S. Walker, Effet de la rotation sur les instabilités thermocapillaires dans un pont liquide chauffé latéralement, Proc. $18^{\mathrm{e}}$ Congrès Français de Mécanique, 2007

[8] J. Herrero, F. Giralt, J.A.C. Humphrey, Influence of the geometry on the structure of the flow between a pair of corotating disks, Phys. Fluids 11 (1999) 88-96

[9] A. Randriamampianina, R. Schiestel, M. Wilson, Spatiotemporal behaviour in an enclosed corotating disk pair, J. Fluid Mech. 434 (2001) 39-64

[10] M. Piva, E. Meiburg, Steady axisymmetric flow in an open cylindrical container with a partially rotating bottom wall, Phys. Fluids 14 (2005) 063603 